\title{
12 Digitale Transformation am Beispiel der Automobilindustrie
}

\author{
T. Riasanow, G. Galic, M. Böhm, H. Krcmar
}

Die Entstehung neuer Services durch digitale Innovationen bedrohen zunehmend existierende Geschäftsmodelle. In der Automobilindustrie werden technologische Trends wie selbstfahrende Autos, Konnektivität und Car-Sharing zahlreiche Möglichkeiten für neue Geschäftsmodelle eröffnen. Diese Technologien schaffen auch Möglichkeiten für innovative neue Marktteilnehmer, welche gerade dabei sind, die Automobilindustrie fundamental zu transformieren. Darum zeigt dieses Kapitel das bestehende Wertschöpfungsnetzwerk der Automobilindustrie basierend auf der e $e^{3}$-value-Modellierungsmethode. Grundlage für das Modell sind 15 generische Rollen und die Wertströme zwischen den Rollen, welche basierend auf den CrunchbaseDaten von 650 Unternehmen in der Automobilindustrie entwickelt wurden. Zur Validierung des Wertschöpfungsnetzwerks wurden fünf Interviews mit Industrieexperten durchgeführt. Die Ergebnisse verdeutlichen die zentrale Rolle von Mobilitätsdienstplattformen, Anbietern disruptiver Technologien und die Verschmelzung unterschiedlicher Branchen, beispielsweise da OEMs mit Anbietern mobiler Zahlungen kollaborieren. Praktiker können das Modell verwenden, um die Wertschöpfung der Automobilindustrie zu analysieren und um sich im Markt zu positionieren oder um mögliche disruptive Akteure und potentielle Geschäftspartner zu identifizieren. ${ }^{34}$

\footnotetext{
${ }^{34}$ Hierbei handelt es sich um die Übersetzung des Artikels „Digital Transformation in the Automotive Industry: Towards a Generic Value Network“ (Riasanow, Galic \& Böhm 2017), präsentiert auf der 25th European Conference on Information Systems (ECIS) in Guimarães, Portugal.
}

(C) Der/die Autor(en) 2018

G. Oswald und H. Krcmar (Hrsg.), Digitale Transformation, Informationsmanagement und digitale Transformation, https://doi.org/10.1007/978-3-658-22624-4_12 


\subsection{Einleitung}

Neue Technologien beschleunigen digitale Innovationen, welche das tägliche Leben von Konsumenten, Unternehmen und die gesamte Struktur eines Ökosystems fundamental transformieren können (Fichman, Dos Santos, \& Zheng, 2014). Heute verändert die digitale Transformation sogar die Wertschöpfung von Industrien, in denen Wert ausschließlich durch physische Produkte erzeugt wird (Yoo, Henfridsson, \& Lyytinen, 2010), was in der Automobilindustrie am deutlichsten sichtbar wird. Digitale Innovationen wie Software für selbstfahrende Autos, Big Data Analytics und soziale Netzwerke revolutionieren die Automobilindustrie grundsätzlich (Gao et al., 2016; Hanelt, Piccinini, Gregory, Hildebrandt, \& Kolbe, 2015; Simonji-Elias et al., 2014; Wijnen, 2013). Etablierte Unternehmen müssen sich des disruptiven Charakters dieser Technologien bewusst sein und ihre Geschäftsmodelle anpassen, um mit den aufstrebenden Akteuren im Ökosystem konkurrieren zu können (Eriksen \& Letiche, 2008; Möller, Legner, \& Heck, 2011; Perrott, 2008).

Mit der Entstehung dieser Technologien werden Plattformen und innovative digitale Dienste von einer Vielzahl neuer Marktteilnehmer wie Tesla, Uber oder ZipCar angeboten, die das etablierte Ökosystem der Automobilindustrie bedrohen (Gao et al., 2016). Die Marktkapitalisierung des prominentesten Beispiels, Uber, der größte Peer-to-Peer (P2P) Online-Vermittlungsdienst zur Personenbeförderung, war bereits im Jahr 2015 höher als 80 \% der S\&P500 Unternehmen, u. a. General Motors und Ford, bewertet (Verhage, 2015). Aufgrund der steigenden Anzahl neuer Marktteilnehmer sind die Automobilhersteller (OEMs) nicht mehr alleine im Markt und müssen ihre Strategien entsprechend anpassen. Vor allem, um gegenüber den neuen Marktteilnehmern, die kundenorientierte Mobilität bieten und damit das bestehende Wertschöpfungsnetzwerk nachhaltig verändern, konkurrenzfähig zu bleiben (Berman \& Bell, 2011; Gao et al., 2016; Matt, Hess, \& Benlian, 2015). Hierfür werden Strategien benötigt, welche den tiefgreifenden Einfluss unternehmensweiter Veränderungen, die durch digitale Technologien induziert werden, widerspiegeln (Chanias \& Hess, 2016). Um auf Trends wie z. B. Car-Sharing oder autonomes Fahren reagieren zu können, müssen Unternehmen ihre Organisationen wandeln und in diesem Zuge auch traditionelle Geschäftsmodelle anpassen, die seit Jahrzehnten etabliert waren (Fitzgerald, Kruschwitz, Bonnet, \& Welch, 2013; Lucas, Agarwal, Clemons, El Sawy, \& Weber, 2013). Derzeit investieren OEMs stark, um die Trends nicht zu verschlafen. Daher ist in den nächsten fünf Jahren wahrscheinlich mit einer Disruption der Geschäftsmodelle zu rechnen (Simonji-Elias et al., 2014). Allerdings bleibt unklar, welche Technologien sich 
durchsetzen werden, was zu Spannungen in der Automobilindustrie führt, da OEMs ihre Führungsposition in Produkt und Technologie nicht aufgeben wollen (Simonji-Elias et al., 2014). Dennoch ist der transformative Einfluss auf Unternehmen, welche primär physische Produkte herstellen, bis vor kurzem kaum in der Praxis beachtet worden (Yoo et al., 2010). Dieser wurde erst im Rahmen der digitalen Transformation von Automobilunternehmen untersucht. Beispielsweise haben Hanelt et al. (2015) vier Typen von Geschäftsmodelltransformationen identifiziert: Erweiterung, Revision, Terminierung und Kreation. Aufbauend auf diesen vier Typen haben wir 27 unterschiedliche Geschäftsmodelle identifiziert, welche Start-Ups im Mobilitätssektor in den letzten zehn Jahren umgesetzt haben. Dennoch fehlt eine ganzheitliche Analyse der aktuellen und laufenden Transformation der Automobilindustrie (Hanelt et al., 2015), da sich bestehende Studien nur mit den Geschäftsmodellen der Unternehmen beschäftigen. Deswegen analysieren wir hier die digitale Transformation der Automobilindustrie aus der ganzheitlichen Perspektive ihres Wertschöpfungsnetzwerks. Der zentrale Vorteil der Darstellung als Wertschöpfungsnetzwerk im Vergleich zu einem Business Model Canvas besteht darin, die Wertströme zwischen allen Akteuren im Netzwerk analysieren zu können. Zur ganzheitlichen Analyse der Automobilindustrie werden folgende Fragen beantwortet:

- Welche generischen Rollen existieren im Wertschöpfungsnetzwerk der Automobilindustrie?

- Wie gestaltet sich das generische Wertschöpfungsnetzwerk der Automobilindustrie?

Zur Beantwortung dieser Fragen haben wir ein e3-value Wertschöpfungsnetzwerk aus 15 generischen Rollen erstellt. Dieses basiert auf der Analyse von 650 Unternehmen anhand von Daten aus Crunchbase. Crunchbase ist eine umfassende Datenbank bestehend aus Daten von Start-Ups und etablierten Unternehmen. ${ }^{35}$

Der Rest dieses Kapitels ist wie folgt aufgebaut. Zuerst analysieren wir im theoretischen Hintergrund die Literatur zu Wertschöpfungsnetzwerken und die digitale Transformation der Automobilindustrie. Zweitens beschreiben wir das das Forschungsvorgehen. Als Drittes werden die abgeleiteten generischen Rollen und das entwickelte Wertschöpfungsnetzwerk vorgestellt. Abschließend werden die Ergebnisse kritisch diskutiert und im Ausblick die Implikationen aufgezeigt.

\footnotetext{
${ }^{35}$ https://www.crunchbase.com/
} 


\subsection{Theoretischer Hintergrund}

Servitisierung ist nach Service-dominanter Logik (S-D-Logik) einer der wichtigsten Trends in einer zunehmend digitalisierten und vernetzten Welt (Lusch \& Nambisan, 2015; Vargo \& Lusch, 2004). Diese Logik impliziert, dass Dienstleistungen in Dienstleistungsökosystemen erstellt werden. In diesen wird Wert nicht mehr nur von einem Akteur geschaffen, sondern zunehmend gemeinsam (sog. co-creation) durch mehrere Beteiligte (Lusch \& Nambisan, 2015).

\subsubsection{Von Wertschöpfungsketten zu Wertschöpfungsnetzwerken}

Der Begriff der Dienstleistungsökosysteme geht auf die Literatur zu Wertschöpfungsketten zurück. Das Konzept der Wertschöpfungsketten wurde in den letzten Jahrzehnten hauptsächlich benutzt um Industrien zu verstehen und zu analysieren (Stabell \& Fjeldstad, 1998). Am bekanntesten ist der Ansatz von Porter (1985). Traditionell werden in der produzierenden Industrie Wertschöpfungsketten für die Visulisierung der verketteten physischen Aktivitäten benutzt (Porter, 1985). Wertschöpfungsketten können ebenfalls benutzt werden um Wettbewerber oder neue Marktteilnehmer zu analysieren (Böhm, Koleva, Leimeister, Riedl, \& Krcmar, 2010; Peppard \& Rylander, 2006). Im Laufe der Zeit hat Porter eine erweiterte Wertschöpfungskette entwickelt, ein Wertschöpfungssystem, das die Wertschöpfungsketten des produzierenden Unternehmens, der Zulieferer und der Kunden einschließt. Dieses System hilft die Interdependenzen zwischen den Akteuren aufzuzeigen. Wertschöpfungssysteme sind entscheidend für Unternehmen, da durch die Optimierung oder Koordination der Verknüpfungen zwischen den Akteuren Wettbewerbsvorteile geschaffen werden können (Connolly \& Matarazzo, 2009). Jedoch ist in einer globalisierten und dynamischen Welt ist der Erklärungsgehalt von Wertschöpfungsketten begrenzt, wodurch eine komplexere Methode erforderlich wurde, die zu Wertschöpfungsnetzwerken führte (Biem \& Caswell, 2008). Nach Peppard und Rylander (2006) versteht man unter einem Wertschöpfungsnetzwerk eine Menge von relativ autonomen Einheiten, die unabhängig verwaltet werden können, aber im Rahmen gemeinsamer Prinzipien und Service Level Agreements (SLAs) zusammenarbeiten. Jeder Akteur trägt einen inkrementellen Wert zum Wertschöpfungsnetzwerk bei (Bovet \& Martha, 2000), konzentriert sich dabei aber nur auf seine Kernkompetenzen (Stabell \& Fjeldstad, 1998). Ein Wertschöpfungsnetzwerk ist auch eine adäquate Methode, um interorganisationale Beziehungen zu visualisieren (Biem \& Caswell, 2008), in dem Funktionen und Aktivitäten, die gleichzeitig durchgeführt werden gezeigt werden können. Der Vorteil des Wertschöpfungsnetzwerks liegt in der Möglichkeit zur Darstellung von Kooperationsbeziehungen und strategischen Allianzen. Durch die zunehmende Komplexität 
von Unternehmensbeziehungen, die durch Digitalisierung hervorgerufen werden, können Branchen nicht mehr ausschließlich als Lieferanten, Kunden und Wettbewerber eingestuft werden (Biem \& Caswell, 2008; Böhm et al., 2010; Peppard \& Rylander, 2006; Pil \& Holweg, 2006). Heute verändert die Digitalisierung auch die Wertschöpfungsnetzwerke von Unternehmen, die physische Güter produzieren. Darum werden Wertschöpfungsnetzwerke nicht mehr nur von dienstleistungsorientierten Industrien benutzt (Peppard \& Rylander, 2006). Durch Digitalisierung verschmelzen die digitale und physische Welt ohnehin (Hanelt et al., 2015). Zusammenfassend verwenden wir den ganzheitlichen Ansatz eines Wertschöpfungsnetzwerks, um die digitale Transformation in der Automobilindustrie zu betrachten, da viele disruptive Innovationen und Veränderungen derzeit in dieser Industrie passieren (Berman \& Bell, 2011; Gao et al., 2016).

\subsubsection{Die Digitale Transformation der Automobilindustrie}

Im Fall der Automobilindustrie fokussiert sich die Literatur auf verschiedene Aspekte der digitalen Transformation, beginnend mit einem Überblick über die verschiedenen Typen von Geschäftsmodellveränderungen (Hanelt et al., 2015) für spezifische Transformationsstrategien. Hanelt et al. (2015) verbinden die Phänomene der digitalen und physischen Welt und erforschen den Einfluss von digitalen Trends auf das Geschäftsmodell von Unternehmen in der Automobilindustrie. Ihre Ergebnisse zeigen vier verschiedene Typen von Geschäftsmodellveränderungen: Erweiterung, Revision, Terminierung und Kreation. Beispiele für die Erweiterung von Geschäftsmodellen sind interaktive Elemente mit den Kunden, z. B. durch soziale Medien. Eine Revision von Geschäftsmodellen erfolgt durch selbstfahrende Autos, welche aus physischen und digitalen Komponenten bestehen. Die Terminierung von Geschäftsmodellen kann durch Virtualisierung erfolgen, z. B. können virtuelle Ausstellungsräume Autohäuser substituieren. Die Kreation von neuen Geschäftsmodellen kann durch neue Fahrdienste oder neue datenbasierte Dienste erreicht werden. Bei der Untersuchung von Strategien für die digitale Transformation haben Chanias und Hess (2016) bestehende Herausforderungen der digitalen Transformation in der Automobilindustrie untersucht. Dafür führten sie eine Fallstudie zur Entwicklung von Strategien nach einem aktivitätsbasierten Prozessmodell durch (Chanias \& Hess, 2016). Ihre Fallstudie zeigt, dass digitale Transformation in erster Linie durch eine Vielzahl von organisatorischen Aktivitäten aus einer Bottom-Up Perspektive beginnt, noch bevor das Top-Management eine ganzheitliche Strategie etabliert hat. Hildebrandt, Hanelt, Firk, und Kolbe (2015) haben festgestellt, dass digitale technologie-bezogene Zusammenschlüsse und Akquisitionen 
(M\&As) einen positiven Einfluss auf digitale Geschäftsmodellinnovationen haben. OEMs haben so die Möglichkeit externes Wissen zu digitalen Innovationen zu erwerben (Henfridsson \& Lind, 2014). Das daraus entstehende digitale Ökosystem um den OEM ist ein wichtiger Erfolgsfaktor für digitale Geschäftsmodelle (Hildebrandt et al., 2015). Die Ergebnisse von Hildebrandt et al. (2015) zeigen, dass Offenheit gegenüber externen Marktteilnehmern und Wissen die Entwicklung von digitalen Innovationen unterstützt (Hildebrandt et al., 2015). Entsprechend der Theorie zu disruptiven Innovationen können digitale Innovationen die Geschäftsleistung erhöhen und zu einem besseren Nutzungserlebnis führen (Keller \& Hüsig, 2009). Da externes Wissen eine wichtige Rolle spielt ist es wichtig das ganze Ökosystem der Automobilindustrie zu untersuchen. Daher führten Piccinini, Hanelt, Gregory, und Kolbe (2015) eine Delphi-Studie mit Industrieexperten durch, um die aufkommenden Herausforderungen, die mit der digitalen Transformation der zumeist physischen Automobilindustrie einhergehen, zu identifizieren. Für digitale Ökosysteme sind unter diesen folgende genannt: Wettbewerb mit einer wachsenden Anzahl von neuen Konkurrenten und industriefremden Rivalen (bspw. IT-Industrie); die Bildung von komplementären Partnerschaften zwischen verschiedenen Ökosystemen (bspw. Business und IT) zur Gestaltung neuer Geschäftsmodelle; die Überbrückung von Lücken zwischen bisher getrennten Unternehmenseinheiten und Teilnehmern im Ökosystem, um neuen, digitalen Wert zu schaffen; die Verbesserung der Informationsflüsse und des Austauschs zwischen Geschäftspartnern im Wertschöpfungsnetzwerk, um ein nahtloses Nutzungserlebnis zu ermöglichen (Piccinini et al., 2015). Ausgehend vom Paradoxon organisatorischer Ambidextrie zeigen sie Organisationen, die gleichzeitig aktuelle Ressourcen ausschöpfen, während sie in neue Fähigkeiten investieren müssen (Gregory, Keil, Muntermann, \& Mähring, 2015). Zuletzt haben Remané, Hildebrandt, Hanelt, und Kolbe (2016) die Geschäftsmodelle von aufstrebenden StartUps im Mobilitätssektor untersucht. Dafür haben sie auf die Crunchbase Datenbank zurückgegriffen, um die Geschäftsmodelle der Start-Ups nach dem Vorgehen von Weill, Malone, D’Urso, Herman, und Woerner (2005) zu klassifizieren. Basierend auf dieser Klassifikation haben sie 27 unterschiedliche Geschäftsmodelle identifiziert, die zu vier Clustern zusammengefasst wurden: Creator, Distributor, Landlord und Broker. Dennoch fehlt der Forschung eine detaillierte Analyse der Akteure der anhaltenden digitalen Transformation der Automobilindustrie (Hanelt et al., 2015), da bisherige Studien sich ausschließlich auf die Geschäftsmodelle der Unternehmen fokussiert haben. So analysieren wir die digitale Transformation der Automobilindustrie aus der ganzheitlichen Perspektive ihres Wertschöpfungsnetzwerks. Um dies zu erreichen, müssen wir die generischen Rollen und Wertströme zwischen den Rollen identifizieren. 


\subsection{Forschungsvorgehen}

Unser Forschungsvorgehen besteht aus drei Schritten. Zuerst haben wir die generischen Rollen für das Wertschöpfungsnetzwerk und die Wertströme zwischen den Rollen identifiziert. Zweitens haben wir das generische Netzwerk anhand der identifizierten Rollen und Wertströme visualisiert. Drittens wurde das Modell mit semi-strukturierten Experteninterviews validiert.

Im ersten Schritt entschieden wir uns, Crunchbase-Daten zu verwenden, um die Rollen im Wertschöpfungsnetzwerk abzuleiten. Crunchbase verfügt über eine umfangreiche Datenbank aus bestehenden Firmen und Start-Ups, einschließlich einer Beschreibung des Wertversprechens der Unternehmen (Marra, Antonelli, Dell'Anna, \& Pozzi, 2015). Dabei aktualisiert Crunchbase kontinuierlich die Informationen zu den jeweiligen Finanzierungsrunden der StartUps (Marra et al., 2015; Perotti \& Yu, 2015). Für diesen Artikel haben wir die gelisteten Crunchbase-Daten am 20. Oktober 2016 extrahiert. Um alle Organisationen der Automobilindustrie sowie verwandte Technologien zu sammeln, haben wir die Crunchbase-Kategorienliste mit dem Suchbegriff ,,automotive“ gefiltert, was zu einer Stichprobengröße von 728 finanzierten Unternehmen führte, zu denen 78 Börsengänge (IPOs) gehören. Wir haben Unternehmen ausgeschlossen, die bisher ,geschlossen“ wurden, z.B. WhipCar, ein in London ansässiger CarSharing-Service. Darüber hinaus mussten wir drei weitere Unternehmen ausschließen, da ihre Website nicht mehr existierte. Bei der Sichtung der Daten haben wir Unternehmen entdeckt, die keine direkte Beziehung zur Automobilindustrie hatten, z. B. Eni, ein Energieunternehmen, das sich mit Öl- und Erdgasförderung und -r-affinerie beschäftigt. Mit den verbleibenden 650 Unternehmen führten wir im ersten Schritt eine strukturierte Inhaltsanalyse durch, einschließlich einer induktiven Kategorisierung auf Basis von Mayring (2010) und Miles und Huberman (1994). Mit dieser Methode haben wir 15 generische Rollen identifiziert. Um eine konsistente Kodierung zu gewährleisten, wurde die Interrater-Reliabilität überprüft. Zwei der Autoren haben die 650 Organisationen dafür unabhängig kodiert. Bevor die Autoren die Unternehmen aus der Crunchbase-Datenbank kodiert haben, haben sie jeweils einige Unternehmen initial geprüft, um sich mit dem Kodierungsschema vertraut zu machen und um anschließend die Ergebnisse der initialen Kodierung für Kalibrierungszwecke zu vergleichen. Alle Autoren bestätigten die endgültige Kodierung jeder Organisation und diskutierten die Diskrepanzen der Kodierungen, bis ein Konsens erreicht wurde. Dies trug erheblich zur Reduzierung der Diskrepanzen in der Kodierung bei (Bullock \& Tubbs, 1990). Zum Beispiel haben wir Vroom basierend auf der Beschreibung "Vroom is an online direct car retailer that makes car-buying and-selling fast and easy” als „Autohändler“ klassifiziert. Der gleiche Ansatz wurde für die Identifizierung der 
Wertströme verwendet, jedoch hier die Crunchbase-Daten mit öffentlich zugänglichen Sekundärdaten wie Unternehmenswebsites, Berichte, Jahresberichte oder Presseartikel verknüpft. Zum Beispiel haben wir die Wertströme zwischen OEMs und Lieferanten als Austausch von Technologie (Hardware und Software) und Geld basierend auf dem Zitat "Continental's five largest OEM customers (Daimler, Fiat-Chrysler, Ford, General Motors, and VW) generated approximately $43 \%$ of the Continental Corporation's sales in 2016" im aktuelle Jahresbericht von Continental klassifiziert (Continental AG, 2016). Nachdem alle Kodierer die Klassifikation abgeschlossen hatten, wurde Krippendorff's (2004) Alpha benutzt, um die die Interrater-Reliabilität zu berechnen. Die Berechnung ergab ein Alpha von 0,87, welches einer akzeptablen Interrater-Reliabilität entspricht (Krippendorff, 2004).

Im zweiten Schritt verwendeten wir die $\mathrm{e}^{3}$-value-Methode, um das Wertschöpfungsnetzwerk der Automobilindustrie anhand der identifizierten generischen Rollen und der Wertströme zwischen den generischen Rollen zu visualisieren. Die $\mathrm{e}^{3}$-value-Methode ist eine Modellierungsmethode, die dabei es ermöglicht, Geschäftsideen aus Perspektive eines Wertschöpfungsnetzwerks darzustellen, zu analysieren und zu bewerten. Die Methode wird insbesondere verwendet, um die ökonomische Nachhaltigkeit von Wertschöpfungsnetzwerken durch die Modellierung des Austauschs von Gütern mit ökonomischen Wert zu bewerten (Gordijn \& Akkermans, 2003).

Im dritten Schritt führten wir fünf Interviews mit Experten aus der Automobilindustrie durch, um das generische Wertschöpfungsnetzwerk zu validieren. Wir haben eine semistrukturierte Interviewtechnik (Myers \& Newman, 2007), um zwei CEOs von "Value Added Partners", einen CEO einer Technologietochter eines OEMs, einen Abteilungsleiter eines weiteren OEMs und einen Senior Consultant mit Fokus auf große IT-Anwendungen der Automobilindustrie zu interviewen. Jeder der Experten konnte mindestens zehn Jahre Erfahrung in der Automobilindustrie vorzuweisen und beschäftigt sich mit neuen digitalen Technologien. Die Befragten befanden sich entweder in einer führenden strategischen Management- oder IT-Position (Goldberg, Satzger, \& Fromm, 2016), die privilegierten Zugang zu Informationen und Wissen über strategische Themen der Automobilindustrie haben (Bogner, Littig, \& Menz, 2009). Das erlaubte uns auf breites Wissen, langjährige Markterfahrung und unterschiedliche Einblicke von verschiedenen Unternehmen aufzubauen. Die Interviews wurden zwischen Dezember 2016 und März 2017 durchgeführt. Jedes der Interviews wurde aufgezeichnet, anschließend transkribiert und dauerte durchschnittlich ca. 41 Minuten. Um die generischen Rollen und Wertströme 
zu validieren, diskutierten wir die Rollen und Wertströme des Wertschöpfungsnetzwerks mit allen Experten.

\subsection{Entwicklung eines generischen Wertschöpfungsnetzwerks für die}

\section{Automobilindustrie}

Aufgrund digitaler Innovationen wandelt sich die Automobilindustrie, welches eine Reihe neuer Marktteilnehmer im Wertschöpfungsnetzwerk entstehen lässt. Nach dem Ansatz von Böhm et al. (2010) abstrahieren wir von Marktteilnehmern oder Akteuren, die ähnliche Angebote für Kunden anbieten und definieren generische Rollen, basierend auf einer strukturierten Inhaltsanalyse der Crunchbase-Daten der 650 identifizierten Unternehmen, siehe Tabelle 7.

Tabelle 7: Generische Rollen der Akteure im Wertschöpfungsnetzwerk der Automobilindustrie (Quelle: Riasanow, Galic, und Böhm (2017))

\begin{tabular}{|c|c|c|}
\hline Rolle & Beschreibung & Beispiel(e) \\
\hline OEM & $\begin{array}{l}\text { Der Original Equipment Manufacturer (OEM) } \\
\text { produziert Autos. OEMs können sowohl traditio- } \\
\text { nelle Verbrennungsmotoren als auch Elektromoto- } \\
\text { ren für Autos produzieren. Die Wertschöpfung von } \\
\text { OEMs kann Direktvertrieb, Produktion, After-Sa- } \\
\text { les, Forschung und Entwicklung und Services be- } \\
\text { inhalten (Kang, Mahoney, \& Tan, 2009). }\end{array}$ & $\begin{array}{l}\text { Ferrari, Tesla, Ca- } \\
\text { dillac, BMW, } \\
\text { Daimler, Bolt Mo- } \\
\text { torbikes }\end{array}$ \\
\hline Kunden & $\begin{array}{l}\text { Kunden fordern Mobilität, die in vielen Formen er- } \\
\text { füllt werden kann: Nutzung des eigenen Fahrzeugs, } \\
\text { Vermittlung und Sharing eines Autos, öffentliche } \\
\text { Verkehrsmittel oder durch bestimmte Mobilitäts- } \\
\text { dienste wie Uber. Verbraucher können Produkte o- } \\
\text { der Dienstleistungen vor, während oder nach dem } \\
\text { Transport verwenden. In einigen Bereichen ist } \\
\text { spricht man von Prosumenten, indem gleichzeitig } \\
\text { ein Dienst eingesetzt und kreiert wird. Ein Beispiel } \\
\text { ist die gemeinsame Nutzung von personenbezoge- } \\
\text { nen Daten via Smartphone mit Google Maps unter } \\
\text { Verwendung der aggregierten Echtzeit-Verkehrs- } \\
\text { informationen anderer Benutzer für die Navigation. }\end{array}$ & \\
\hline
\end{tabular}




\begin{tabular}{|c|c|c|}
\hline & $\begin{array}{l}\text { Verbraucher können für Dienstleistungen mit Geld, } \\
\text { persönlichen Daten oder einer Kombination von } \\
\text { beidem bezahlen. }\end{array}$ & \\
\hline $\begin{array}{l}\text { Tier -1-3- } \\
\text { Lieferanten }\end{array}$ & $\begin{array}{l}\text { Die traditionelle Automobilindustrie zeichnet sich } \\
\text { durch eine einseitige Lieferanten-Käufer-Bezie- } \\
\text { hung aus (Turnbull, Oliver, \& Wilkinson, 1992). } \\
\text { Fahrzeughersteller verlassen sich stark auf Tier-1- } \\
\text { Lieferanten, die etwa } 85 \% \text { der Teile liefern. Tier- } \\
\text { 1-Lieferanten können Produktentwicklung, Design } \\
\text { und Technologie anbieten und hängen zumeist von } \\
\text { weiteren Zulieferern ab (sog. Tier-2- oder Tier-3- } \\
\text { Lieferanten). }\end{array}$ & $\begin{array}{l}\text { Bosch, Continental, } \\
\text { Faurecia, China } \\
\text { Automotive Sys- } \\
\text { tems, ABC Group }\end{array}$ \\
\hline $\begin{array}{l}\text { Öffentliche } \\
\text { Verkehrsmittel }\end{array}$ & $\begin{array}{l}\text { Diese Rolle repräsentiert den traditionellen öffent- } \\
\text { lichen Verkehr, einschließlich U- und Straßenbah- } \\
\text { nen, Bussen und Zügen (Hoffmann, Ipeirotis, \& } \\
\text { Sundararajan, 2016). }\end{array}$ & $\begin{array}{l}\text { New York MTA, } \\
\text { citibike }\end{array}$ \\
\hline Autovermietung & $\begin{array}{l}\text { Diese Rolle bietet verschiedene Modelle für die } \\
\text { Anmietung eines Autos an (Moeller \& } \\
\text { Wittkowski, 2010). }\end{array}$ & Sixt, Hertz \\
\hline $\begin{array}{l}\text { Auto-/Ersatz- } \\
\text { teilehändler }\end{array}$ & $\begin{array}{l}\text { Bei dieser Rolle können - abgesehen von einigen } \\
\text { OEMs - Autos oder Autoteile direkt erworben } \\
\text { werden. Autos und Ersatzteile können auch über } \\
\text { Online-Plattformen der jeweiligen Händler ver- } \\
\text { kauft werden (Applegate, 2000). }\end{array}$ & $\begin{array}{l}\text { LUEG, Amazon } \\
\text { (Fiat), carparts.com }\end{array}$ \\
\hline $\begin{array}{l}\text { Anbieter } \\
\text { disruptiver } \\
\text { Technologie }\end{array}$ & $\begin{array}{l}\text { Disruptive Technologieanbieter bieten disruptive } \\
\text { Innovationen für OEMs in Form von Hardware } \\
\text { und Software an, bspw. Sensoren für autonomes } \\
\text { Fahren. Nach Christensen (1997) können disrup- } \\
\text { tive Technologien etablierten Technologien zu Be- } \\
\text { ginn unterlegen sein.Durch rapides Wachstum }\end{array}$ & $\begin{array}{l}\text { Savari, Intel, Mo- } \\
\text { bileye }\end{array}$ \\
\hline
\end{tabular}




\begin{tabular}{|c|c|c|}
\hline & $\begin{array}{l}\text { können disruptive Technologien bald zu einer Be- } \\
\text { seitigung oder Ersetzung von etablierten Techno- } \\
\text { logien führen (Christensen, 1997). }\end{array}$ & \\
\hline $\begin{array}{l}\text { Mobilitäts- } \\
\text { dienstleistungs- } \\
\text { plattform }\end{array}$ & $\begin{array}{l}\text { Wir unterscheiden zwischen verschiedenen Mobi- } \\
\text { litätsdienstleistungs-Plattformen wie privaten oder } \\
\text { kommerziellen Car-Sharing, P2P-Lending oder } \\
\text { Service-Plattformen von OEMs (Lee, Chan, } \\
\text { Balaji, \& Chong, 2016). Mobilitätsdienste können } \\
\text { über diese Plattformen abgerufen werden, z. B. } \\
\text { bietet Uber eine Plattform, die Fahrern ermöglicht } \\
\text { ihren Mobilitätsdienst für registrierte Nutzer zur } \\
\text { Verfügung zu stellen. }\end{array}$ & $\begin{array}{l}\text { Uber, VRide, } \\
\text { DriveNow, } \\
\text { Tesloop, Taxify, } \\
\text { Car2Go }\end{array}$ \\
\hline $\begin{array}{l}\text { Mobilitäts- } \\
\text { dienstleistungs- } \\
\text { aggregator }\end{array}$ & $\begin{array}{l}\text { Diese Rolle aggregiert verschiedene Mobilitäts- } \\
\text { dienstleistungen, einschließlich öffentlicher Ver- } \\
\text { kehrsdienste und Car-Sharing-Plattformen, die } \\
\text { auch intermodale Mobilitätsdienste beinhalten } \\
\text { können (Plummer \& Kenney, 2009). }\end{array}$ & Moovel, Flare \\
\hline $\begin{array}{l}\text { Anbieter } \\
\text { intelligenter } \\
\text { Infrastruktur }\end{array}$ & $\begin{array}{l}\text { Diese Rolle stellt den Zusammenhang von physi- } \\
\text { scher und digitaler Infrastruktur dar. Aufgrund der } \\
\text { Konnektivität neuer Technologien, z.B. Sensoren } \\
\text { und Elektrofahrzeuge, kann Infrastruktur, z. B. } \\
\text { Verkehrszeichen oder Parkplätze mit Autos und } \\
\text { Verbrauchern verbunden werden. Ein Beispiel da- } \\
\text { für sind Ladestationen für Elektroautos, deren Zu- } \\
\text { stand online abgerufen werden kann. }\end{array}$ & $\begin{array}{l}\text { ChargeNow, Car- } \\
\text { Charging, Charger- } \\
\text { link }\end{array}$ \\
\hline $\begin{array}{l}\text { Cloud- } \\
\text { Infrastruktur- } \\
\text { Anbieter }\end{array}$ & $\begin{array}{l}\text { Ein Cloud-Infrastruktur-Anbieter (IaaS) besteht } \\
\text { aus einem gemeinsamen Pool von Internet-basier- } \\
\text { ten Rechenressourcen (z.B. Server, Speicher), die } \\
\text { mit minimalen Managementaufwand schnell be- } \\
\text { reitgestellt und freigegeben werden können } \\
\text { (Youseff, Butrico, \& Da Silva, 2008). }\end{array}$ & $\begin{array}{l}\text { Amazon Elastic } \\
\text { Compute Cloud } \\
\text { (Amazon EC2) }\end{array}$ \\
\hline
\end{tabular}




\begin{tabular}{|c|c|c|}
\hline $\begin{array}{l}\text { Cloud-Plattfor- } \\
\text { manbieter }\end{array}$ & $\begin{array}{l}\text { Ein Cloud-Plattform-Anbieter (PaaS) bietet einen } \\
\text { digitalen Marktplatz für verschiedene Cloud-Infra- } \\
\text { struktur-Dienste an. Ziel ist es, Kunden und } \\
\text { Dienstleister zu vernetzen. Erstere können nach } \\
\text { geeigneten Diensten suchen, während der Dienst- } \\
\text { leister seine Dienstleistungen anbieten kann. Die } \\
\text { Plattform basiert auf der zugrundeliegenden } \\
\text { Cloud-Infrastruktur (Youseff et al., 2008). }\end{array}$ & $\begin{array}{l}\text { Google Cloud Plat- } \\
\text { form, Microsoft } \\
\text { Azure }\end{array}$ \\
\hline $\begin{array}{l}\text { Anbieter von } \\
\text { Mehrwertdiens- } \\
\text { ten }\end{array}$ & $\begin{array}{l}\text { Mehrwertdienste können vor, während oder nach } \\
\text { dem Transport abgerufen werden. Es gibt zwei } \\
\text { Arten von Mehrwertdiensten (SaaS). Erstere sind } \\
\text { Telematik-Dienste oder technische Informationen } \\
\text { über das Fahrzeug, Sicherheitsmerkmale oder in- } \\
\text { telligente Fahrassistenzsysteme. Weitere bieten } \\
\text { Kunden Entertainment, Sicherheit, standortbezo- } \\
\text { gene Informationsdienste oder Concierge-Ser- } \\
\text { vices. Diese Dienste können über Cloud-Plattfor- } \\
\text { men aufgerufen werden (Youseff et al., 2008). }\end{array}$ & $\begin{array}{l}\text { Spotify, Data } \\
\text { Crossover, Au- } \\
\text { tolinked, ParkNow, } \\
\text { OnStar, BMW } \\
\text { Connected Drive }\end{array}$ \\
\hline $\begin{array}{l}\text { Auto- } \\
\text { dienstleistungen }\end{array}$ & $\begin{array}{l}\text { Autodienstleistungen umfassen alle klassischen } \\
\text { Dienstleistungen wie Wartung, Versicherung rum } \\
\text { um das Auto oder stationäre Dienste wie Autowä- } \\
\text { sche Remané et al. (2016). }\end{array}$ & Washtec \\
\hline $\begin{array}{l}\text { Anbieter von } \\
\text { Online- } \\
\text { Zahlungen }\end{array}$ & $\begin{array}{l}\text { Diese Rolle ermöglicht Bezahlungen, die auch auf } \\
\text { mobilen Endgeräten durchführbar sind. }\end{array}$ & MercedesPay \\
\hline
\end{tabular}

Da sich unsere Rollen auf einem abstrakteren Level als Geschäftsmodelle befinden, kann sich eine Rolle auf mehrere Geschäftsmodelltypen beziehen. Des Weiteren kann ein Unternehmen in verschiedenen Rollen vorkommen, indem es unterschiedliche Dienste anbietet. Die identifizierten generischen Rollen wurden mit den Geschäftsmodelltypen von Remané et al. (2016) verglichen. Wir fanden für jeden Geschäftsmodelltyp ein Beispiel in unseren Rollen. Beispielsweise haben wir aufgrund der ähnlichen Wertströme im Wertschöpfungsnetzwerk die Geschäftsmodelltypen „Digital Service Provider“ und „Sensor-enabled Service Innovator“ zur 
generischen Rolle „Anbieter von Mehrwertdiensten“ aggregiert. Zweitens wurde die e3-valueMethode benutzt, um ein generisches Wertschöpfungsnetzwerk für die Automobilindustrie zu erstellen, das in Abbildung 34 dargestellt ist. Es zeigt die identifizierten Rollen und Wertströme zwischen ihnen, die wir durch die fünf Experteninterviews validiert haben. Rollen können Wert durch die Bereitstellung von Daten, Diensten oder physischen Produkten (Auto, Mietwagen, Ersatzteile, Technologie etc.) erzeugen. Durch Informationen auf öffentlich verfügbaren Webseiten der Unternehmen haben wir folgende Wertströme in Bezug auf Dienstleistungen identifiziert: Car as a Service (CaaS), Software as a Service (SaaS) und Mobility as a Service (MaaS).

Darüber hinaus wurde die Cloud-Computing Perspektive nach Böhm et al. (2010) im Wertschöpfungsnetzwerk aufgegriffen, welche zwischen Platform as a Service (PaaS) und Infrastructure as a Service (IaaS) unterscheidet. Die Service-Ebene des Cloud-Computing (SaaS) wurde hier als Value Added Services bezeichnet, z. B. BMW Connected Drive. Produkte und Dienstleistungen werden entweder gegen Geld, Daten oder einer Kombination von beiden ausgetauscht. Im Mittelpunkt des Wertschöpfungsnetzwerks steht der Kunde, welcher MaaS, CaaS oder SaaS verlangt, oder ein physisches Produkt (Auto, Mietwagen, etc.) kauft oder mietet.

Das generische Wertschöpfungsnetzwerk zeigt, dass sich die Automobilindustrie in Richtung eines mehrseitigen Markts verändert und sich vom klassischen Lieferanten-Käufer-Geschäftsmodell zunehmend entfernt. Anknüpfend an die Ergebnisse von Remané et al. (2016) können wir bestätigten, dass neue Rollen wie „Mobilitätsdienstleistungsplattform“ oder „Anbieter intelligenter Infrastrukturen“ im Netzwerk entstehen. Basierend auf den Ergebnissen des erstellten Wertschöpfungsnetzwerks bedrohen neu entstehende Rollen die Wertschöpfung der OEMs von zwei Seiten. Einerseits gibt es Mobilitätsdienstleistungsplattformen wie Uber, die den direkten Kontakt zum Kunden über die eigene Plattform hergestellt haben und den Kunden somit direkt Services anbieten können. OEMs verlieren also zunehmend den Kontakt zum Kunden. Andererseits zwingen Trends, wie selbstfahrende Autos, die OEMs zu Kooperationen mit neu entstehenden Rollen. Dies wird durch die zentrale Rolle der Anbieter disruptiver Technologie zwischen OEMs und Mobility Services im Wertschöpfungsnetzwerk dargestellt. Beispiele hierfür sind Mobileye und Intel, welche mit BMW kooperieren um selbstfahrende Autos zu realisieren. Daher müssen OEMs offen für neue Marktteilnehmer sein und externe Kenntnisse erlangen um Innovationen zu fördern (Hildebrandt et al., 2015). Die Öffnung nach außen ist auch besonders wichtig um das Nutzungserlebnis zu verbessern, beispielsweise durch komplementäre Apps (Keller \& Hüsig, 2009). 


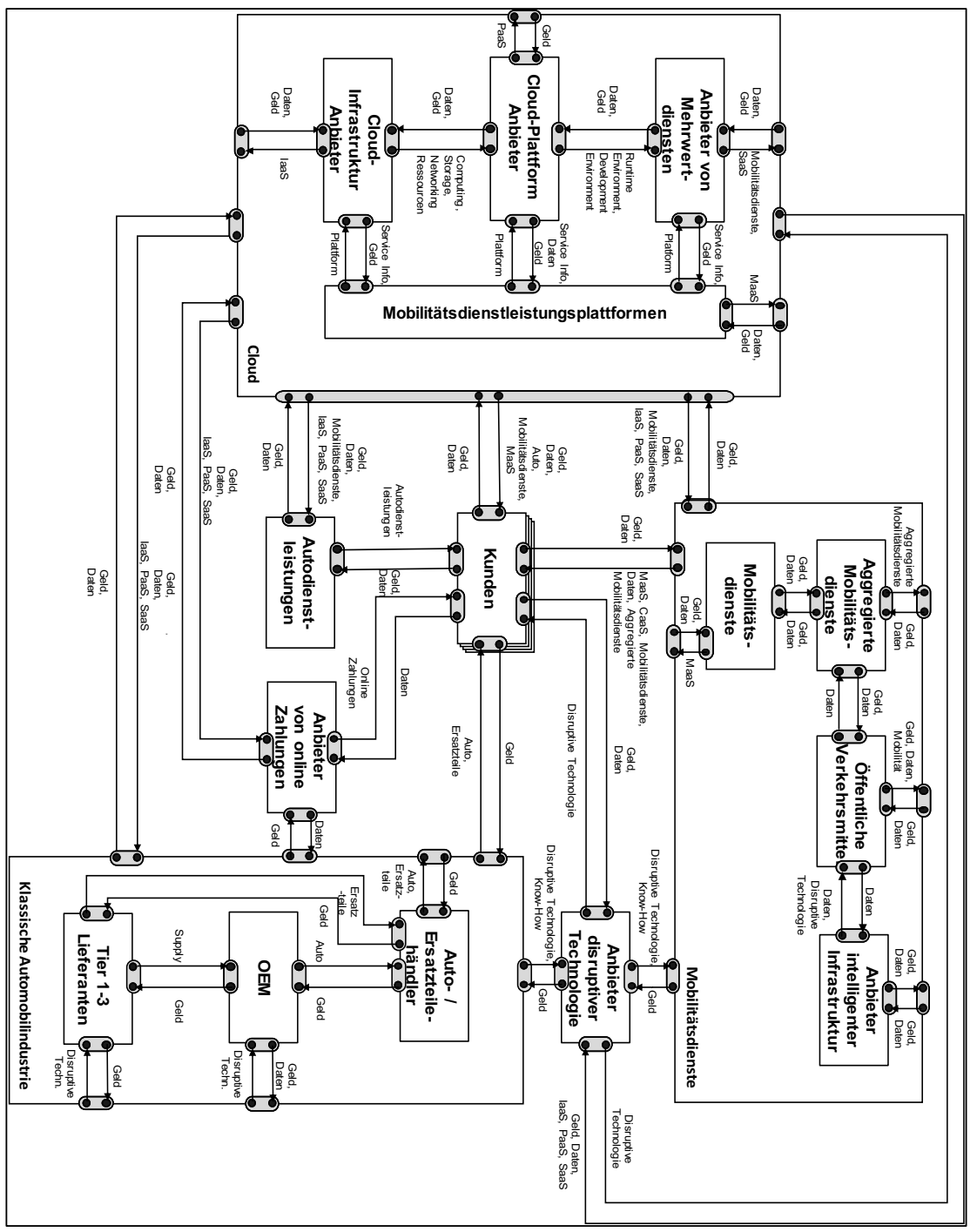

Abbildung 34: Generisches Wertschöpfungsnetzwerk der Automobilindustrie (Quelle: Riasanow et al. (2017))

Das generische Wertschöpfungsnetzwerk unterstreicht die Erstellung neuer datengestützter Rollen durch digitale Innovationen, z. B. als Anbieter intelligenter Infrastruktur, die in Echtzeit Verkehrsdaten mit autonomen Autos teilen. Die Experteninterviews zeigten die zunehmende Verbreitung von Mobilitätsdienstleistungen durch digitale Technologien, da einer der Experten 
vorschlug, die Rolle des Anbieters von Online-Zahlungen im Netzwerk aufzunehmen. So erwarb zum Beispiel Daimler einen elektronischen Zahlungsdienstleister für mobile Zahlungen, der nun als „MercedesPay“ für Mobilitätsdienste wie Car2Go oder als virtuelle Brieftasche für Kunden dient (Daimler AG, 2017).

\subsection{Zusammenfassung und Ausblick}

Dieses Kapitel stellt ein generisches Wertschöpfungsnetzwerk für die Automobilindustrie vor, das auf 15 generischen Rollen basiert, die durch eine strukturierte Inhaltsanalyse der Crunchbase-Daten von 650 Automobil-Organisationen identifiziert wurden. Das entwickelte Modell zeigt, dass die digitale Transformation neue Rollen im Wertschöpfungsnetzwerk der Automobilindustrie schafft. Zudem zeigt es, dass Mobilitätsdienstplattformen und Anbieter disruptiver Technologie den Markt durchdringen und damit die Wertschöpfung von OEMs von zwei Seiten gleichzeitig bedrohen.

Das Modell ist durch die Informationen der Crunchbase-Datenbank und unsere Kodierung der generischen Rollen limitiert. Für die Entwicklung der Wertströme zwischen den Rollen stützten wir uns auf öffentlich verfügbare Informationen wie Firmenwebsites, Berichte, Presseartikel oder Jahresabschlussberichte der Unternehmen. Darüber hinaus führten wir semistrukturierte Interviews mit Experten aus der Automobilindustrie durch, um das vorgeschlagene generische Wertschöpfungsnetzwerk zu validieren.

Das entwickelte Wertschöpfungsnetzwerk kann für weitere Analysen im Kontext digitaler Transformation verwendet werden. Praktiker, z.B. OEMs, können das Modell verwenden, um potentielle Bedrohungen für ihre aktuelle Marktposition, neue Möglichkeiten zur Anpassung an Trends oder die Veränderung von Kundenbedürfnissen zu identifizieren. Als Beispiel haben innovative OEMs wie BMW mit DriveNow frühzeitig eine Car-Sharing Plattform eingeführt. Auch Mercedes hat vor kurzem einen mobilen Zahlungsdienstleister erworben, um das digitale Portfolio zu erweitern (Daimler AG, 2017). Zusammengefasst können Unternehmen das generische Wertschöpfungsnetzwerk benutzen, um ihre eigene Position in der Automobilindustrie zu analysieren und ihre Beziehungen zu Partnern oder Wettbewerbern zu bewerten. Nach (Böhm et al., 2010) ist es nicht zwingend notwendig zu wissen, welche Rolle den größten Anteil innerhalb des Wertschöpfungsnetzwerks einnehmen könnte, sondern ein Alleinstellungsmerkmal basierend auf den eigenen Kernkompetenzen zu entwickeln. 


\subsection{Literaturverzeichnis}

Applegate, L. M. (2000). E-Business Models: Making Sense of the Internet Business Landscape, Upper Saddle River, NJ.

Berman, S. J., \& Bell, R. (2011). Digital Transformation: Creating New Business Models where Digital Meets Physical: IBM Institute for Business Value.

Biem, A., \& Caswell, N. (2008). A Value Network Model for Strategic Analysis. Vorgestellt auf: 41st Hawaii International Conference on System Science, Waikoloa, HI, USA.

Bogner, A., Littig, B., \& Menz, W. (2009). Introduction: Expert Interviews - An Introduction to a New Methodological Debate. In: A. Bogner, B. Littig, \& W. Menz (Hrsg.), Interviewing Experts (S. 1-13). London, UK: Palgrave Macmillan.

Böhm, M., Koleva, G., Leimeister, S., Riedl, C., \& Krcmar, H. (2010). Towards a Generic Value Network for Cloud Computing. In: J. Altmann \& O. F. Rana (Hrsg.), Economics of Grids, Clouds, Systems, and Services. GECON 2010. Lecture Notes in Computer Science (Band 6296). Berlin, Heidelberg, Deutschland: Springer.

Bovet, D., \& Martha, J. (2000). Value Nets: Breaking the Supply Chain to Unlock Hidden Profits. New York, NY, USA: John Wiley \& Sons.

Bullock, R., \& Tubbs, M. E. (1990). A Case Meta-Analysis of Gainsharing Plans as Organization Development Interventions. The Journal of Applied Behavioral Science, 26(3), 383-404. doi:10.1177/0021886390263011

Chanias, S., \& Hess, T. (2016). Understanding Digital Transformation Strategy Formation: Insights from Europe's Automotive Industry. Vorgestellt auf: 20th Pacific Asia Conference on Information Systems, Chiayi, Taiwan.

Christensen, C. M. (1997). The Innovator's Dilemma: When New Technologies Cause Great Firms to Fail. Boston, MA: Harvard Business School Press.

Connolly, S., \& Matarazzo, J. (2009). Knowledge and Special Libraries. London, UK: Routledge.

Continental AG. (2016). Annual Report 2016. URL: http://www.continentalcorporation.com/www/download/portal_com_en/themes/ir/financial_reports/downloa d/annual report 2016 en.pdf

Daimler AG. (2017). ePayment-Investition: Daimler Financial Services übernimmt PayCash Europe SA. URL: http://media.daimler.com/marsMediaSite/ko/de/15310294

Eriksen, M., \& Letiche, H. (2008). Leading adaptive organizational change: self-reflexivity and self-transformation. Journal of Organizational Change Management, 21(5), 622640. doi:10.1108/09534810810903252 
Fichman, R. G., Dos Santos, B. L., \& Zheng, Z. (2014). Digital Innovation as a Fundamental and Powerful Concept in the Information Systems Curriculum. MIS Quarterly, 38(2), 329-343.

Fitzgerald, M., Kruschwitz, N., Bonnet, D., \& Welch, M. (2013). Embracing Digital Technology: A New Strategic Imperative. MIT Sloan Management Review, 1.

Gao, P., Müller, N., Kaas, H.-W., Mohr, D., Wee, D., Hensley, R., . . Kohler, D. (2016). Automotive Revolution Rerspective Towards 2030. URL: https://www.mckinsey.de/files/automotive revolution_perspective towards_2030.pdf.

Goldberg, M., Satzger, G., \& Fromm, H. (2016). Adapting IT Service Management For Successful Multi-Sourcing Service Integration. Vorgestellt auf: 24th European Conference on Information Systems, İstanbul, Türkei.

Gordijn, J., \& Akkermans, J. (2003). Value-based Requirements Engineering: Exploring Innovative E-commerce Ideas. Requirements Engineering, 8(2), 114-134.

Gregory, R. W., Keil, M., Muntermann, J., \& Mähring, M. (2015). Paradoxes and the Nature of Ambidexterity in IT Transformation Programs. Information Systems Research, 26(1), 57-80.

Hanelt, A., Piccinini, E., Gregory, R. W., Hildebrandt, B., \& Kolbe, L. M. (2015). Digital Transformation of Primarily Physical Industries-Exploring the Impact of Digital Trends on Business Models of Automobile Manufacturers. Vorgestellt auf: Wirtschaftsinformatik, Osnabrück, Deutschland.

Henfridsson, O., \& Lind, M. (2014). Information Systems Strategizing, Organizational Subcommunities, and the Emergence of a Sustainability Strategy. Journal of Strategic Information Systems, 23(1), 11-28. doi:10.1016/j.jsis.2013.11.001

Hildebrandt, B., Hanelt, A., Firk, S., \& Kolbe, L. (2015). Entering the Digital Era - The Impact of Digital Technology-related M\&As on Business Model Innovations of Automobile OEMs. Vorgestellt auf: 36th International Conference on Information Systems, Fort Worth, TX, USA.

Hoffmann, K., Ipeirotis, P., \& Sundararajan, A. (2016). Ridesharing and the Use of Public Transportation. Vorgestellt auf: Proceedings of the 37th International Conference on Information Systems, Dublin, Ireland.

Kang, M. P., Mahoney, J. T., \& Tan, D. (2009). Why Firms make Unilateral Investments Specific to Other Firms: The Case of OEM Suppliers. Strategic Management Journal, 30(2), 117-135. doi:10.1002/smj.730

Keller, A., \& Hüsig, S. (2009). Ex Ante Identification of Disruptive Innovations in the 
Software Industry Applied to Web Applications: The Case of Microsoft's vs. Google's Office Applications. Technological Forecasting and Social Change, 76(8), 10441054.

Krippendorff, K. (2004). Content Analysis: An Introduction to Its Methodology. Thousand Oaks, CA: Sage.

Lee, Z. W., Chan, T. K., Balaji, M., \& Chong, A. Y.-L. (2016). Technology-Mediated Sharing Economy: Understanding User Participation in Collaborative Consumption Through the Benefitcost Perspective. Vorgestellt auf: 20th Pacific Asia Conference on Information Systems, Chiayi, Taiwan.

Lucas, H. C., Agarwal, R., Clemons, E. K., El Sawy, O. A., \& Weber, B. W. (2013). Impactful Research on Transformational Information Technology: An Opportunity to Inform New Audiences. MIS Quarterly, 37(2), 371-382.

Lusch, R. F., \& Nambisan, S. (2015). Service Innovation: A Service-Dominant Logic Perspective. MIS Quarterly, 39(1), 155-175.

Marra, A., Antonelli, P., Dell'Anna, L., \& Pozzi, C. (2015). A network analysis using metadata to investigate innovation in clean-tech - Implications for energy policy. Energy Policy, 86, 17-26. doi:10.1016/j.enpol.2015.06.025

Matt, C., Hess, T., \& Benlian, A. (2015). Digital Transformation Strategies. Business \& Information Systems Engineering, 57(5), 339-343.

Mayring, P. (2010). Qualitative Inhaltsanalyse. Wiesbaden, Germany: VS Verlag für Sozialwissenschaften.

Miles, M. B., \& Huberman, A. M. (1994). Qualitative Data Analysis: An Expanded Sourcebook. Newbury Park, CA: Sage.

Moeller, S., \& Wittkowski, K. (2010). The Burdens of Ownership: Reasons for Preferring Renting. Managing Service Quality: An International Journal, 20(2), 176-191. doi:doi.org/10.1108/09604521011027598

Möller, D., Legner, C., \& Heck, A. (2011). Understanding IT Transformation - An Explorative Study. Vorgestellt auf: 19th European Conference on Information Systems, Helsinki, Finnland.

Myers, M. D., \& Newman, M. (2007). The qualitative interview in IS research: Examining the craft. Information and Organization, 17(1), 2-26. doi:10.1016/j.infoandorg.2006.11.001

Peppard, J., \& Rylander, A. (2006). From Value Chain to Value Network: Insights for Mobile Operators. European Management Journal, 24(2), 128-141. 
Perotti, V., \& Yu, Y. (2015). Startup Tribes: Social Network Ties That Support Success in New Firms. Vorgestellt auf: 21st Americas Conference on Information Systems, Fajardo, Puerto Rico.

Perrott, B. E. (2008). Towards a Model of Transformation: Manager's Perceptions of Transformation in an E-business Environment. Journal of Information \& Knowledge Management, 7(2), 63-74.

Piccinini, E., Hanelt, A., Gregory, R., \& Kolbe, L. (2015). Transforming Industrial Business: The Impact of Digital Transformation on Automotive Organizations. Vorgestellt auf: 36th International Conference on Information Systems, Fort Worth, TX, USA.

Pil, F. K., \& Holweg, M. (2006). Evolving from Value Chain to Value Grid. Sloan Management Review, 47(4), 72-80.

Plummer, D. C., \& Kenney, L. F. (2009). Three Types of Cloud Brokerages will Enhance Cloud Services.

Porter, M. E. (1985). Competitive Advantage: Creating and Sustaining Superior Performance. New York, NY: FreePress.

Remané, G., Hildebrandt, B., Hanelt, A., \& Kolbe, L. M. (2016). Discovering New Digital Business Model Types - A Study of Technology Startups from the Mobility Sector. Vorgestellt auf: 20th Pacific Asia Conference on Information Systems, Chiayi, Taiwan.

Riasanow, T., Galic, G., \& Böhm, M. (2017). Digital Transformation in the Automotive Industry: Towards a Generic Value Network. Vorgestellt auf: 25th European Conference on Information Systems, Guimarães, Portugal.

Simonji-Elias, M., Collyer, M., Johnston, M., Lichtenfeld, L., Lund, J., \& Staiger, J. (2014). KPMG's Global Automotive Executive Survey 2014. URL:

https://www.kpmg.com/DE/de/Documents/global-automotive-executive-survey-2014KPMG.pdf.

Stabell, C. B., \& Fjeldstad, Ø. D. (1998). Configuring Value for Competitive Advantage: on Chains, Shops, and Networks. Strategic Management Journal, 19(5), 413-437.

Turnbull, P., Oliver, N., \& Wilkinson, B. (1992). Buyer-supplier relations in the UK automotive industry: Strategic implications of the Japanese manufacturing model. Strategic Management Journal, 13(2), 159-168. doi:10.1002/smj.4250130207

Vargo, S. L., \& Lusch, R. F. (2004). Evolving to a New Dominant Logic for Marketing. Journal of Marketing, 68(1), 1-17. doi:10.1509/jmkg.68.1.1.24036

Verhage, J. (2015). Uber Is Now Valued Higher Than $80 \%$ of the Companies in the S\&P 500 . 
URL: https://www.bloomberg.com/news/articles/2015-12-03/uber-s-latest-fundinground-gives-it-a-larger-valuation-than-80-percent-of-the-s-p-500

Weill, P., Malone, T. W., D’Urso, V. T., Herman, G., \& Woerner, S. (2005). Do Some

Business Models Perform Better than Others? A Study of the 1000 Largest US Firms.

MIT Center for Coordination Science.

Wijnen, R. (2013). New Business Models in the Car Industry. URL:

http://www.businessmodelsinc.com/new-business-models-in-the-carindustry/\#sthash.MEkq2LL8.dpuf.

Yoo, Y., Henfridsson, O., \& Lyytinen, K. (2010). Research Commentary-The New

Organizing Logic of Digital Innovation: An Agenda for Information Systems

Research. Information Systems Research, 21(4), 724-735. doi:10.1287/isre.1100.0322

Youseff, L., Butrico, M., \& Da Silva, D. (2008). Toward a Unified Ontology of Cloud

Computing. Vorgestellt auf: 2008 Grid Computing Environments Workshop, Austin, TX, USA.

Open Access Dieses Kapitel wird unter der Creative Commons Namensnennung 4.0 International Lizenz (http://creativecommons.org/licenses/by/4.0/deed.de) veröffentlicht, welche die Nutzung, Vervielfältigung, Bearbeitung, Verbreitung und Wiedergabe in jeglichem Medium und Format erlaubt, sofern Sie den/die ursprünglichen Autor(en) und die Quelle ordnungsgemäß nennen, einen Link zur Creative Commons Lizenz beifügen und angeben, ob Änderungen vorgenommen wurden.

Die in diesem Kapitel enthaltenen Bilder und sonstiges Drittmaterial unterliegen ebenfalls der genannten Creative Commons Lizenz, sofern sich aus der Abbildungslegende nichts anderes ergibt. Sofern das betreffende Material nicht unter der genannten Creative Commons Lizenz steht und die betreffende Handlung nicht nach gesetzlichen Vorschriften erlaubt ist, ist für die oben aufgeführten Weiterverwendungen des Materials die Einwilligung des jeweiligen Rechteinhabers einzuholen. 